\title{
„Wir leben sterbend“ (Lukas Bärfuß) \\ Über eine Spielart des Heroischen im Zeichen des Sisyphos
}

\author{
Magnus Striet
}

\section{Heroismen unter den Bedingungen nachmetapybischen Denkens}

In einer berühmten Passage der Götzen-Dämmerung heißt es bei Friedrich Nietzsche: „Die wahre Welt haben wir abgeschafft: welche Welt blieb übrig? die scheinbare vielleicht? ... Aber nein! mit der wahren Welt haben wir auch die scheinbare abgeschafft! ${ }^{{ }^{1}}$ Die aus der späten Schaffensphase Nietzsches stammende Aufhebung von wahrer und scheinbarer Welt bildet einen nochmaligen Reflex auf die Rede vom Tode Gottes. Als Nietzsche in der Fröblichen Wissenschaft seinen letzten Menschen den Tod Gottes als irreversibles geschichtliches Ereignis ausrufen ließ ${ }^{2}$ und dieser die um ihn Herumstehenden dazu aufforderte, endlich einzusehen, dass der alte Gott tot sei und die Kirchen nur noch die Grabmäler des verwesenden Gottes seien, reagierte er auf einen ideengeschichtlichen Umwälzungsprozess, der sich im europäischen Kulturkontext bereits seit geraumer Zeit ankündigte.

Die Gründe dafür kann ich nicht in ihrer ganzen Komplexität rekonstruieren. ${ }^{3}$ Weil es mir hier um die Frage geht, welche Bedeutung dieser Umwälzungsprozess für das Phänomen von Heroisierungs- beziehungsweise Selbstheroisierungsprozessen hat, präziser noch: wie im geschichtlichen Prozess generierte Heldenfigurative dekonstruiert werden und es im historischen Vergleich zu neuen, ganz und gar nicht heldischen Helden kommt, konzentriere ich mich auf zwei Gründe, die folgend noch expliziter ausgeführt werden: Zum einen geriet der Willkürgott des Augustinus in die Krise, weil er aus moralischen Gründen nicht mehr akzeptiert wurde, zum anderen gewann die theologisch abgedrängte Theodizeefrage wieder neu an Bedeutung. Diese Gründe sind deshalb zu erinnern, weil sie bei dem Heldentypus, um den es mir geht, wirksam sind. Meine grundlegende These lautet: Nur wenn man den ideengeschichtlichen Umwälzungsprozess in den Hintergrund nimmt, bekommt man bestimmte, erst in einer metaphysisch und religiös haltlos gewordenen Moderne möglich gewordene (Selbst-)Heroisierungsphänomene in den Blick.

1 Friedrich Nietzsche: Götzen-Dämmerung (KSA 6), München 21988, S. 55-161, hier S. 81, Hervorhebung im Original. Der Vortragsstil wurde für die Publikation beibehalten.

2 Vgl. Friedrich Nietzsche: Die fröhliche Wissenschaft (KSA 3), München 2 1988, S. 343-651; S. 480-481.

3 Vgl. ausführlicher Magnus Striet: In der Gottesschleife. Von religiöser Sehnsucht in der Moderne, Freiburg 22015. 
Die europäische Moderne ist nicht ohne den bereits erwähnten Nietzsche zu verstehen. ${ }^{4}$ Wie neu sein Gedankengut war oder ob er nicht vor allem ein Katalysator einer sich seit geraumer Zeit ankündigenden Umstellung des Weltbildes war, das nach einer anderen als der aus bisher geltenden metaphysisch-theologischen Gedankenbeständen abgesicherten Beschreibung des Menschen verlangt, sei dahingestellt. Nietzsche beobachtete bezogen auf die europäischen Kontexte präzise, wie die über lange historische Zeiträume selbstverständlich akzeptierte Geltung eines weitgehend christlich-religiös codierten Weltbildes in der Auflösung begriffen war. Der augustinische Willkürgott mit seinem doppelten Prädestinationsgehabe war unter Verdacht gestellt, ethischen Ansprüchen nicht zu genügen. Das die gesamte Menschheit in eine massa damnata versammelnde Erbsündenkonstrukt war ohnehin unter den Verdacht mangelnder intellektueller Stichhaltigkeit gestellt worden. Hinzu kam das bohrende Theodizeeproblem, das zwar über Jahrhunderte hinweg durch eine das gesamte anthropologische Denken dominierende Sündenrhetorik stillgestellt werden konnte, dann aber zumindest in Intellektuellenkreisen mit dem ausgehenden 18. Jahrhundert eine neue Präsenz gewann. Angesichts des Elends, welches die Pestwellen über Europa brachten, wurde das Sich-Verschweigen Gottes zum Stachel im Fleisch des Gottglaubens. Als Kant dann noch die Unmöglichkeit eines jeden Gottesbeweisversuchs nachwies und er die endlich-praktische Vernunft als die unhintergehbare Prüfinstanz etablierte, in der entschieden werden muss, was ethisch geboten ist ${ }^{5}$-, war es um den die Individuen und Gesellschaften stabilisierenden Faktor Gott geschehen. Soziologisch gesehen lässt sich hier sicherlich kein einliniger Prozess identifizieren, und auch lässt sich nach Kant beobachten, wie man nochmals zur alten, wenn auch neu begründeten Metaphysik zurückzukehren versuchte. ${ }^{6}$ Die ideengeschichtliche europäische Moderne ist von Ungleichzeitigkeiten, von Homogenisierungs- und zugleich wieder von Pluralisierungsdynamiken bestimmt. Aber nicht zu übersehen ist, dass es mit der anwachsenden Religionskritik aus den oben ausgeführten Gründen eine in Intellektuellenkreisen verbreitete melancholische Unruhe gibt, die dergestalt in einer Sinnkrise mündet, als alte historisch generierte Sinnangebote nicht mehr funktionieren.

Mit dieser Unruhe ist nicht der Bereich der Moralität und der Ethik angesprochen. Die Umstellung eines religiös-metaphysischen Naturrechts auf ein Vernunftrecht war nicht nur überfällig, weil das Naturrecht seine begründungslogische Basis verlor, sondern vor allem um der Freiheit und der Möglichkeit von Moralität willen geboten. ${ }^{7}$ Delikat war vielmehr, dass mit dem Wegfall der gro-

Vgl. Andreas Urs Sommer: Nietzsche und die Folgen, Stuttgart 2017.

5 Zur Wirkungsgeschichte Kants vgl. Herbert Schnädelbach: Wir Kantianer. Der „kritische Weg“ heute, in: Deutsche Zeitschrift für Philosophie 55, 2005, S. 835-850.

6 Hier immer noch aufschlussreich Walter Schulz: Der Gott der neuzeitlichen Metaphysik, Pfullingen ${ }^{2} 1959$, S. 89-113.

7 Vgl. Axel Honneth: Das Recht der Freiheit. Grundriß einer demokratischen Sittlichkeit, Berlin 2011, hier S. 38. Höchst interessant wäre es, eine Genealogie moderner Heroismen 
ßen heilsgeschichtlichen Erzählung die Notwendigkeit des Menschen, sich überhaupt eine Geschichte über die Welt und das Mensch genannte, nicht festgestellte Tier erzählen zu müssen, immer noch Bestand hatte. Es ist die eigentümliche, in seinem Selbst-Verhältnis gründende Distanz des Menschen zu sich selbst, die ihn dazu nötigt, sich Geschichten über sich zu erzählen und sich so auszudeuten. Schärfer noch: Angesichts der Gewissheit seines Todes scheint dies eine anthropologische Notwendigkeit zu sein. Mit einem anthropologischen Essentialismus hat dieser Verdacht einer Erzählnotwendigkeit nichts zu tun. Es ist lediglich das Faktum der Bewusstseinsspaltung, welches das Mensch genannte Tier zu einem erzählpflichtigen Tier macht. Faktisch. Was aber bedeutet dies für die Frage nach möglichen Heroisierungsprozessen in einer solchen Moderne?

Heroisierungsprozesse sind eingebettet in sozial geteilte Erzählprozesse. Mythen, religiöse Erzählungen etc. etablieren allererst die wirksame Möglichkeit, andere Personen oder aber sich selbst zu heroisieren. Sie können deshalb nicht begriffslos sein. Nur deshalb kann es im historischen Prozess zu semantischen Umbesetzungen bis dato wirksamer Heldenfigurative kommen. Verlieren religiöse Narrative ihre interpretierende Wirksamkeit, so treten andere an ihre Stelle. Der Verzicht auf eine religiöse oder mythologische Weltausdeutung setzt lediglich eine neue Weltausdeutung frei und so impliziert der Verlust einer von der Vorstellung eines Telos geleiteten Geschichtsphilosophie eine Philosophie der Geschichte. Nietzsche hat ein Heroisierungskonzept etablieren wollen, an dem er aber wohl selbst gescheitert ist. „Wie trösten wir uns, die Mörder aller Mörder“, 8 hatte er den letzten Menschen in der Fröblichen Wissenschaft fragen lassen, nachdem er gerade zuvor diesen noch den Tod Gottes hatte verkünden lassen. Nietzsche wusste noch, was am Gottesglauben hängt. Sein Gegenprogramm gegen das Bewusstsein, ungetröstet bleiben zu müssen, lautete: ,amor fati“ “. ${ }^{9}$ Geschichtsphilosophisch ausgedrückt heißt das bei ihm: Die Welt ist so zu affirmieren, wie sie ist, ohne Abzug und „ohne ein Finale ins Nichts. “10 Aber selbst wenn Geschichte so ausgedeutet wird, die Welt keine Rücksicht auf den Menschen und deshalb die Geschichte kein auf den Menschen bezogenes Telos kennt, handelt es sich um eine Philosophie der Geschichte.

im Medium einer Rekonstruktion dieses Umstellungsprozesses zu schreiben. In den Blick genommen werden müssten dann Figuren wie Hannah Arendt.

8 Nietzsche: Die fröhliche Wissenschaft (Anm. 2), S. 481. Das Projekt einer philosophischen Anthropologie, dessen Ansatzpunkt bei der Beschreibung des Menschen als des nicht festgestellten Tieres einsetzt, ist hier nicht eigens rekonstruiert. Zu seiner Wirkmächtigkeit vgl. Hans Blumenberg: Beschreibung des Menschen. Aus dem Nachlaß hg. von Manfred Sommer, Berlin 2006.

9 Nietzsche: Die fröhliche Wissenschaft (Anm. 2), S. 276.

10 Friedrich Nietzsche: Nachgelassene Fragmente Sommer 1886 - Herbst 1887 (KSA 12), München 21988, hier S. 213. Zu Nietzsches Versuch einer Überwindung des Nihilismus und dessen Konzeption(en) des Heroischen vgl. Christoph Schweer: Heimweh - Heros Heiterkeit. Nietzsches Weg zum Überhelden, Würzburg 2018. 
Nietzsches Geschichtsphilosophie kennt allerdings nicht mehr die Unterscheidung zwischen der scheinbaren und der wahren Welt, von der alle platonisch infizierten Philosophien lebten, aber: Nietzsche kommt nicht von der Bewusstseinsspaltung los, sich immer nicht nur in einer theoretischen Weise konzeptionell zur Welt und zu sich verhalten zu müssen, sondern auch in einer selbstexplikativen normativen Weise und daran scheitert sein geschichtsphilosophisches Gedankenexperiment. Bis in die späten, radikal das reine Diesseits affirmierenden Texte hinein ist zu beobachten, wie diese immanent an der Denkfigur einer unbedingten, vorbehaltlosen Affirmation der Welt, so wie sie ist, und d. h. „ohne Abzug $^{\text {"11 }}$ scheitern. Dies gilt auch für die, die an ihn anschlossen, wie Ernst Jünger mit seinem Konzept des heroischen Realismus. ${ }^{12}$ Ontogenetisch bedingt und kulturhistorisch differenziert scheint der Mensch sich in einer normativ bestimmenden, Alternativen der Selbstausdeutung auslotenden Weise zu sich selbst in ein Verhältnis bringen zu müssen. Das gilt nicht nur rein formal. Menschen werden subjektiviert durch das, was gesellschaftlich und kulturell an Weltausdeutungen, Normativitäten, kulturellen Praxen wirksam ist. Und wirksam sind immer Ideale, die zur Nachahmung auffordern. Deshalb gibt es keine Gesellschaft, die nicht ihre Helden hervorbrächte.

Nietzsche dürfte erahnt haben, dass es den europäisch sozialisierten Menschen kaum möglich sein würde, sich von Gott, präziser: sich von den mit dem Namen Gottes verbindenden Erlösungssehnsüchten zu lösen. Die Grammatik, in der dieser denkt, ist infiziert vom Nicht-Einverstanden-Sein mit dem Tod. Der Tod bleibt auch da noch ein Skandal, wo die Überzeugungskraft religiöser Bewältigungsstrategien längst ihre Glaubwürdigkeit eingebüßt hat. Was bedeutet dies aber für die Heroisierungsfragen? Prägt ein vom geschichtsphilosophischen Ereignis des Todes Gottes bestimmtes Modernebewusstsein neue Heldentypen aus? Folgt man Maurice Merleau-Ponty, so werden alte Heldennarrative lediglich durch neue ersetzt: „Der Held unserer Zeit ist weder ein Skeptiker noch ein Dilettant, noch ein Dekadent. Er hat einfach nur die Erfahrung des Zufalls, der Unordnung und des Scheiterns. ${ }^{\text {13 }}$ Merleau-Ponty schreibt ganz unter dem Eindruck der Katastrophen des 20. Jahrhunderts, aber: Eingebettet ist seine normativ entschiedene, von einem Kantischen Moraltyp bestimmte neue Figuration des Heldischen in eine ideengeschichtliche Rekonstruktion des Dekonstruktionsprozesses geschichtstheologischer beziehungsweise geschichtsphilosophischer Großerzählungen. Nur eine am Ende doch von einem Restessentialismus bestimmte Theorie des Heroischen wird über-

11 Friedrich Nietzsche: Nachgelassene Fragmente Frühjahr - Sommer 1886 (KSA 13), München ${ }^{2} 1988$, hier S. 492.

12 Ernst Jünger: Der heroische Realismus, in: Die literarische Welt 6.13, 1930, 28. März 1930, S. 3-4. Zu Jünger vgl. Dirk Blotzheim: Ernst Jüngers „Heldenehrung“. Zu Facetten in seinem Frühwerk, Oberhausen 2000, hier bes. S. 104-110.

13 Maurice Merleau-Ponty: Der Held, der Mensch (1946), in: ders., Sinn und Nicht-Sinn (Übergänge 35). Aus dem Französischen von Hans-Dieter Gondek, Paderborn 2000, S. 249-255, hier S. 255. 
sehen können, dass jede Zeit ihre Helden kennt. Der von Merleau-Ponty identifizierte neue Typ des Helden lässt sich jedenfalls bis in die jüngste Gegenwart hinein verfolgen.

\section{Melancholisches Berwusstsein und müde Helden}

Nicht das Konzept des heroischen Realismus, für das im 20. Jahrhundert insbesondere der bereits erwähnte Ernst Jünger steht, soll hier Gegenstand werden, sondern das eines melancholischen Heroismus. Die Feuilletonredaktion der Süddeutschen Zeitung hatte jüngst Texte erbeten, die sich allesamt mit der Frage beschäftigten: „Welcher Satz der deutschsprachigen Erzählliteratur ist Ihnen besonders wichtig?"“ Abgedruckt wurde unter anderem ein Text des Schweizerischen Schriftstellers Lukas Bärfuß, der einen Satz von Georg Büchner aus dessen Erzählung Lenz aufnimmt:

„So lebte er hin.“ In diesem gewaltigsten und gleichzeitig gefährlichsten Schlusssatz der Weltliteratur, dem letzten in Büchners Erzählung, spiegeln sich so viele Fragen, dass einem schwindelig wird. Man könnte gut und gern ein paar Jahre, vielleicht ein ganzes Leben, mit seinen Erläuterungen verbringen und würde an kein Ende kommen.

Hier zunächst nur dies: Niemand weiß genau, was dieser Satz aussagt. Der Duden verzeichnet zwei ganz gegensätzliche Bedeutungen des seltenen Verbes „hinleben“. Einerseits „dahinleben“, also ohne Ziel sein; anderseits genau das Gegenteil: „sein Leben auf ein bestimmtes Ziel ausrichten“. Auch das Grimmsche Wörterbuch bringt keine Lösung, im Gegenteil. Zuerst führt es Belege auf für „weiter leben, verleben“, schließlich als zweite Bedeutung auch hier der Gegensatz: „durch Leben zu Grunde richten“" - was nur auf den ersten Blick seltsam erscheint, benutzen wir doch die Wendung „hin sein“ im Sinne von tot, kaputt, zerstört.

Um Büchners Satz eindeutig auszulegen, fehlt eine Präposition. Entweder „Er lebte auf etwas hin“, oder „Er lebte vor sich hin.“ Dieses Fehlen, dieses Schwanken zwischen zwei Bedeutungen, spiegelt eine existenzielle Erfahrung. Wir haben ein Ziel und finden keines. Wir leben und nähern uns dem Tod. Wir leben sterbend. Der Wahnsinn des Helden Lenz wird mit diesem letzten Satz zur Empfindung des Lesers. Wie Lenz bleiben wir ungetröstet, schwankend und am Ende unerlöst. ${ }^{14}$

Das sich hier ausdrückende melancholische Bewusstsein zeigt exakt den ideengeschichtlichen Umbruch auf, den ich versuchte anzudeuten. Der alte Gott mit seinem Erlösungsversprechen ist als der tote Gott noch lebendig. Das trostbedürftige Tier weiß darum, ungetröstet bleiben zu müssen, denn: Von Nietzsches Heroisierung derer, die nur auf sich gestellt das heilige Ja zu allem zu sprechen imstande sind und darin ein unbeugsames Glück empfinden, ist hier nichts mehr zu spüren. Es ist vielmehr der Verzicht „auf Hoffnung und die eigensinnige Bekundung eines

14 Lukas Bärfuß: Und es war alles, alles gut, Süddeutsche Zeitung, 29. Dezember 2017, https:// www.sueddeutsche.de/kultur/letzte-saetze-und-es-war-alles-allesgut-1.3809059, 7. November 2018. 
Lebens ohne Trost", wie ihn Albert Camus ausgesprochen hat. ${ }^{15}$ Ist dies noch Geschichtsphilosophie? Ja - aber eine, die kein Telos kennt, das über ein selbstverantwortetes Ziel innerhalb des Bewusstseinsraumes, der durch das Absurde abgesteckt ist, hinausginge. Damit ist zugleich angedeutet, dass das Absurde selbst nicht einfach ist, so wie die Welt als solche nicht absurd ist. Es existiert vielmehr menschlicher Geist, so dass die als absurd bezeichnete Welt als diese nur im menschlichen Geist existiert. ${ }^{16}$ Was aber folgt daraus für die Heroisierungsthematik?

Dass man sich Sisyphos als einen „glücklichen Menschen vorstellen“17 müsse, bildet die Schlusscoda des Mythos von Sisyphos, und in diesem Sinn ist für Camus Sisyphos der „Held des Absurden“. ${ }^{18}$ In Die Pest spielt Camus dann ein mögliches ethisches Selbstverständnis im Angesicht des Absurden durch. Hier ist der Held der Arzt Rieux, der allerdings nicht mehr vom Glück des Absurden spricht, sondern nur noch müde ist angesichts des sinnlosen Sterbens an der Pest. Dabei steht die Pest, wie Camus in seinen Tagebüchern einmal vermerkt hat, für das Leben selbst. Rieux ist in seinem berechtigten Gottesfuror ein ethisch entschiedener, von eigener Schulderfahrung bedrückter und zutiefst melancholischer Held.

In einer nicht mehr religiös codierten Moderne muss das Sterben anders semantisiert werden. Bezogen auf die Heldenthematik ist nicht entscheidend, ob das in historischen Heroisierungsprozessen generierte Vokabular ausdrücklich angewendet, es neu mit Bedeutung versehen wird, sondern ob es bezogen auf diese Modernitätserfahrungen neue Helden gibt, die eine Identifikation erlauben beziehungsweise zur Nachahmung auffordern. Ich will dies an zwei Beispielen aus der Literatur erörtern. Dabei gehe ich davon aus, dass Literatur, um kulturell funktionieren zu können, einen existierenden Resonanzboden findet. Wenn Philosophie ihre Zeit auf den Begriff bringt, so ist Literatur, ja vielleicht sogar die Kunst überhaupt, ihrer Zeit voraus; aber sie kann dies nur, wenn sie die Unruhe ihrer eigenen Zeit erspürt.

Ich möchte zunächst auf einen eher unbekannten Schriftsteller zu sprechen kommen, auf Markus Werner. Einer der intimsten Kenner seines Werkes hat die Figuren, die in dessen Werk erscheinen, wie folgt charakterisiert:

Immer wieder stossen die Helden - es sind ja bei Markus Werner allesamt sehr unheldische Helden, für die die Rilkesche Formel gilt: Wer spricht vom Siegen? Überstehen ist alles - immer wieder also stossen diese Helden auf eine Formulierung, eine Wendung, ein Schlagwort, dass ihnen - eben - schlagartig klarmacht: Diese Welt ist unhaltbar. ${ }^{19}$

15 Albert Camus: Der Mythos von Sisyphos. Ein Versuch über das Absurde. Übers. von Hans-Georg Brenner und Wolfdietrich Rasch. Mit einem kommentierenden Essay von Liselotte Richter, Düsseldorf 1956, hier S. 54. Vgl. ebd., S. 31.

17 Ebd., S. 101.

18 Ebd., S. 99.

19 Martin Ebel: Rede auf Markus Werner zur Verleihung des ProLitteris-Preises 2016, Hundertvierzehn, https://www.hundertvierzehn.de/artikel/rede-auf-markus-werner-zur-verleihung-desprolitteris-preises-2016-\%E2\%80\%93-von-martin-ebel_1734, 7. November 2018. 
Die Figuren von Werner sind allesamt nicht in der Lage, ihr Leben zu bewältigen, weil es nicht zu bewältigen ist. Sie sind verstrickt in ihre Biographien. Die Liebe ist ihnen unmöglich geworden, und: Alles bekommt unendliches Gewicht und wird dadurch noch unerträglicher.

Dem Thema des Sterbens widmet sich Werner immer wieder, am eindringlichsten in dem zuerst 1992 veröffentlichten Roman Bis bald. Der Protagonist hier, Lorenz Hatt, wie immer ein mit seinem Leben Hadernder, erleidet auf einer Auslandsreise einen Herzinfarkt, kommt zurück nach Deutschland, unterwirft sich der üblichen Therapiemaschinerie, findet auch zurück in den Alltag - bis er dann den nächsten Infarkt erleidet, sodass klar ist, er wird nur noch längere Zeit leben können, wenn er transplantiert wird. Die Wartezeit auf das Organ beginnt, alles dreht sich nur noch um die Frage, wann es soweit sein wird, und dann entscheidet er sich, wie aus einer Eingebung heraus, dazu, sich doch nicht transplantieren zu lassen. Ich zitiere eine längere Passage:

Im Bus, ich habe irgendwann den Bus genommen, nicht das Taxi, sind mir die Menschen menschlich vorgekommen, den Jungen habe ich ihr Jungsein, ihr stenographisches Gemunkel nicht verübelt, sogar das plattgedrückte Zirpen aus ihren Walkmans ist mir nicht sehr zur Last gefallen, und die entmutigten, grobkörnigen Gesichter der Älteren und Alten habe ich gemocht. Ein Hündlein hat meine Schuhe abgeschnuppert, dann mein Geschlecht, und eine junge Frau, sie saß mir gegenüber auf der Längsbank, hat ihre Augen geschlossen, vielleicht im Wunsch, sich unsichtbar zu machen. Und dadurch ist ihr Mund besonders ausgesetzt gewesen, ein schöner, ein bildschöner Mund, schwermütig, rein, ich kann ihn nicht beschreiben, ich habe geschaut und geschaut. ${ }^{20}$

Unser Held, Lorenz Hatt, bittet seine im Übrigen als sehr liebenswert geschilderte Haushälterin Frau Guhl noch, ihm „bei der Rückverwandlung“ seines „Wartezimmers in eine gute Stube gelegentlich zu helfen", er lässt sich von der Transplantationsliste streichen, hört Musik und beschreibt sich im letzten Satz des Romans als „gelöst“. 21

Ich hatte die These vertreten, dass in allen Heroisierungsprozessen Geschichtsphilosophien wirksam sind. In der Romanwelt von Markus Werner ist dies eindeutig der Fall. Es sind kulturell wirksam gewordene Religionsbestände, die erinnert werden, aber: Sie haben ihre Plausibilität verloren. Die Erfahrung der ,jüdischchristlichen Tradition, in welcher immer dort, wo Elend ist, die Gnade des Allmächtigen gleichsam im Startloch sitzt" ${ }^{\text {" } 22}$ wird zwar lakonisch erinnert, aber so recht glauben mag man an diese nicht mehr. Sie hat sich aufgezehrt, es gilt sich zum Faktum dessen zu verhalten, sterben zu müssen, ohne auf Religionsplausibilitäten zurückgreifen zu können. Man erklärt Gott zwar auch nicht für tot, aber es bleibt die Unwahrscheinlichkeit. Lorenz Hatt findet zurück ins Leben, indem er sich mit dem Sterben abfindet. Es ist die Aura einer gelassenen Heiterkeit, mit der Werner seinen Helden angesichts des unvermeidlich Kommenden umgibt.

20 Markus Werner: Bis bald. Roman, Frankfurt am Main ${ }^{5}$ 2011, hier S. 235-236.

21 Ebd., S. 236.

22 Ebd., S. 102. 
Ich komme zu meinem zweiten Beispiel, Robert Gernhardt. Seine Lyrik hat sich millionenfach verkauft. Dies ist nicht notwendig ein Zeichen für Qualität, die ich aber bei Gernhardt überhaupt nicht in Abrede stelle. Der ökonomische Erfolg könnte darauf hindeuten, dass sich in dieser Lyrik eine Sicht auf den Menschen darbietet, die Resonanz findet in der Gegenwart. Gernhardt hat eine Darmtumordiagnose gestellt bekommen und er hat den Prozess seiner Krankheit sowie der Therapien mit Gedichten kommentiert. In den späten Gedichten Gernhardts findet sich zwar keine Heldenterminologie, aber das Ich, das hier spricht, heroisiert sich sehr wohl - wenn auch nicht freiwillig. Der kommende Tod ist eine Katastrophe. Robert Gernhardt ist hier ein getreuer Nachfahr Heinrich Heines. Und das Siechtum, der körperliche Verfall ist unerträglich. Wie bereits bei Markus Werner zu beobachten, reflektiert das Ich in diesen Gedichten seine Situation im Medium des Gottglaubens. Gernhardt variiert Strophen eines Gedichts seines FastNamensvetters, Paul Gerhardt, der ganz in der Tradition der lutherischen Orthodoxie, aber auch in der der Physikotheologie dichtet. ${ }^{23}$ Ich zitiere einige Strophen des Gedichts, das Gernhardt dann melancholisch-resigniert umarbeiten wird, so dass es zu einem Gedicht einer an der Gottesfrage zermürbten Moderne wird.

Geh aus / mein hertz / und suche freud

In dieser lieben sommerzeit

An deines Gottes Gaben:

Schau an der schönen gärten zier,

Und siehe / wie sie mir und dir

Sich ausgeschmücket haben.

\section{(...)}

Die glucke führt ihr völklein aus /

Der storch baut und bewohnt sein Haus /

Das schwälblein speist die jungen /

Der schnelle hirsch / das leichte reh

Ist froh / und kömmt aus seiner höh

Ins tiefe graß gesprungen.

(...)

Erwähle mich zum Paradeis und laß mich bis zur letzten Reis

an Leib und Seele grünen, so will ich dir und deiner Ehr allein und sonsten keinem mehr hier und dort ewig dienen. ${ }^{24}$

23 Dazu Wolfgang Philipp: Das Werden der Aufklärung in theologiegeschichtlicher Hinsicht, Göttingen 1957; Amme-Charlott Trepp: Von der Glückseligkeit alles zu wissen. Die Erforschung der Natur als religiöse Praxis in der Frühen Neuzeit, Frankfurt am Main 2009.

24 Paul Gerhardt: Geistliche Lieder. Hg. von Dorothea Wendebourg in Zusammenarbeit mit Andreas Stegmann, Stuttgart 2013, hier S. 95-99. 
Gernhardt schreibt das Gedicht um, präziser: Das lyrische Ich, das in Gernhardts Gedicht seine Situation während der aufgrund einer Tumorerkrankung notwendig geworden Chemotherapie reflektiert, tut dies zwar im Medium des noch ganz im Christentumsnarrativ sich reflektierenden lyrischen Ichs des Paul Gerhardt-Gedichts. Es streicht aber das theologische, die Theodizeefrage entlastende Narrativ von der Sünde des Menschen, das seit den Zeiten des Augustinus bestimmend geworden war. So kehrt es zurück in die ältere, biblische Hiobtradition. Es ist aber insofern radikal modern, als es die Theodizeefrage anschärft und darüber den Gottglauben verabschiedet. Zumindest wird in den historisch gewachsenen Glauben an den Gott Israels und damit des Gottes Jesu eine enorme Fraglichkeit eingezeichnet. Soll hier von Selbstheroisierungsprozessen geredet werden, so nehmen sie die Gestalt an, dass das Ich lieber trostlos mit sich allein bleibt, als sich noch mit einem Gott zu belasten, der sich lebenspraktisch ohnehin als irrelevant erwiesen hat. Der Glaube an den wirkmächtigen, präziser müsste man wohl sagen: wirkwilligen Gott wird zutiefst erschüttert durch dessen empirische Abwesenheit. Robert Gernhardt kennt die Traditionen, in denen dieser Gott geglaubt wurde und wird. Interessant ist, wie diese immer noch wirksam sind, das Bewusstsein bestimmen - allerdings dem lyrischen Ich, das hier spricht und das Gernhardt mit sich selbst identifiziert, nicht mehr existentiell übersetzbar sind.

Geh aus mein Herz

Oder Robert Gernhardt liest Paul Gerhardt während der Chemotherapie

Geh aus mein Herz und suche Leid

in dieser schönen Sommerszeit

an deines Gottes Gaben.

Schau an der schönen Gifte Zier

und siehe, wie sie hier und mir

Sich aufgereihet haben.

Die Bäume stehen voller Laub.

Noch bin ich Fleisch, wann werd ich Staub?

Ein Bett ist meine Bleibe.

Oxaliplatin, Navoban,

die schauen mich erwartend an:

Dem rücken wir zuleibe.

(...)

Die Glucke führt ihr Völkchen aus.

Der Mensch verfällt im Krankenhaus

ganz lärmbedingtem Grimme.

Des Baggers Biß, der Säge Zahn,

die hören sich viel lauter an

als jede Vogelstimme.

(...)

Der Weizen wächset mit Gewalt.

Ich aber fühl mich dürr und alt,

das Weh verschlägt mirs Loben 


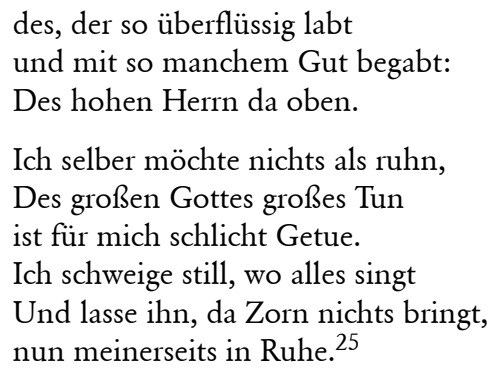

Spricht sich in diesen Versen eine Form von Heroismus aus? Wird hier Selbstheroisierung im Wissen um das unvermeidlich Kommende betrieben? Und worin besteht das Heroische, wenn es sich hier um einen Heroismus handeln sollte?

Anthropologische Begriffe und damit normative Idealvorstellungen werden im geschichtlichen Prozess bestimmt. In der aus religiös-metaphysischen Sinnverheißungen gelösten, des Gottzutrauens aus Theodizeegründen verlustig, deshalb melancholisch gewordenen Moderne sind die alten, geschichtsphilosophische oder gar -theologische Ideen verfolgenden Heroen müde geworden. Auch die reine Größe der Tat des heroischen Realismus macht in dieser Moderne keine Helden mehr; zumal für diese Moderne der Primat einer Ethik steht, die an einem unbedingten und universalen Menschenwürdebegriff orientiert ist. Soll deshalb in einer Theorie des Heroischen ,Außerordentlichkeit ${ }^{26}$ ein Kriterium darstellen in der Frage, ob ein Mensch als Held bezeichnet werden darf, so besteht diese in dieser Moderne lediglich in der Art und Weise, wie das Unvermeidliche ausgehalten wird. Aus der existentiellen Gewissheit heraus, ungetröstet bleiben zu müssen, werden neue Helden geboren. Diese Helden sind in Anlehnung an Max Weber Effekt einer religiös-metaphysischen Unmusikalität. ${ }^{27}$ Unwillentlich ernüchtert leben zu müssen, ist die Geburtsstunde nichtheldischer Helden, die sich aber durchaus selbst als Helden heroisieren und als solche identifiziert werden können. Wer Albert Camus' Mythos von Sisyphos liest, kann sich einen Begriff davon machen, warum dies so ist.

25 Robert Gernhardt: Gesammelte Gedichte. 1954-2006, Frankfurt am Main 62008, hier S. 939-940.

26 Vgl. dazu Tobias Schlechtriemen: Der Held als Effekt. Boundary Work in Heroisierungsprozessen, in: Berliner Debatte Initial 29, 2018, S. 106-119, hier S. 110.

27 Im Anschluss an Max Weber: Wissenschaft als Beruf. Mit zeitgenössischen Resonanzen und einem Gespräch mit Dieter Henrich, hg. von Matthias Bormuth, Berlin 2018, S. 88. 